\title{
CHARACTERIZATION OF CENTRAL ELEMENTS BY INEQUALITIES
}

\author{
AN.AN. NOVIKOV, O.E. TIKHONOV
}

\begin{abstract}
Kazan Federal University, Kremlievskaia st. 18, 420008, Kazan, Russia e-mail: a.hobukob@gmail.com, oleg.tikhonov@kpfu.ru

keywords: von Neumann algebra, $\mathrm{C}^{*}$-algebra, center of algebra, characterization, inequalities. subclass: 46L05, 46L10, 47C15.

Partially supported by Russian Foundation for Basic Research, Grant 14-01-31358.
\end{abstract}

Abstract: We propose a list of inequalities which characterize central elements in von Neumann algebras and $\mathrm{C}^{*}$-algebras.

In this paper we study the possibility to distinguish central elements of operator algebras among all positive elements, by satisfying certain inequalities for positive functionals. A dual problem of characterizing tracial property by inequalities was considered, for instance, in [1, 2, 3, 4, 6, 8, 10, 12, and we apply some machinery of those papers.

Throughout the note, $\mathcal{M}$ stands for a von Neumann algebra, $\mathcal{M}^{s a}$, $\mathcal{M}^{+}$, and $\mathcal{M}^{\text {pr }}$ denote the selfadjoint part, the positive part, and the set of all projections in $\mathcal{M}$, respectively. $\mathcal{Z}$ denotes the center of $\mathcal{M}$ and 1 denotes the identity operator. Let $\mathcal{M}_{*}$ denote the space of all normal functionals on $\mathcal{M}, \mathcal{M}_{*}^{h}$ and $\mathcal{M}_{*}^{+}$denote its Hermitian and positive parts. We will use standard notation for multiplication of a functional $\varphi$ by an operator $x$, namely, $x \varphi, \varphi x$ and $x \varphi x$ denote the linear functionals $y \mapsto \varphi(x y), y \mapsto \varphi(y x)$ and $y \mapsto \varphi(x y x)$, respectively. Recall that a selfadjoint unitary operator in a Hilbert space is said to be a symmetry.

The proof of the following lemma is adapted from [10, Lemma 1].

Lemma 1. Let $a \in \mathcal{M}^{+}$. If the inequality $\varphi($ sas $)<\varphi(a)$ holds for some symmetry $s \in \mathcal{M}$ and some positive normal functional $\varphi \in \mathcal{M}_{*}^{+}$, then there exist positive normal functionals $\psi_{1}, \psi_{2} \in \mathcal{M}_{*}^{+}$, such that $\left|\psi_{1}-\psi_{2}\right|(a)>\psi_{1}(a)+\psi_{2}(a)$.

Proof. For $\lambda>0$ define normal functionals $\psi_{1}^{\lambda}=\lambda s \varphi s+\varphi s+s \varphi+\lambda^{-1} \varphi$ and $\psi_{2}^{\lambda}=\lambda s \varphi s-\varphi s-s \varphi+\lambda^{-1} \varphi$. Since $\psi_{1}^{\lambda}=\lambda v_{1}^{\lambda} \varphi v_{1}^{\lambda}$ with $v_{1}^{\lambda}=$ $s+\lambda^{-1} \mathbf{1}$ and $\psi_{2}^{\lambda}=\lambda v_{2}^{\lambda} \varphi v_{2}^{\lambda}$ with $v_{2}^{\lambda}=s-\lambda^{-1} \mathbf{1}$, those functionals are positive.

Let us demonstrate that $\left|\psi_{1}^{\lambda}-\psi_{2}^{\lambda}\right|=2 \varphi+2 s \varphi s$. Clearly, $\mid \psi_{1}^{\lambda}-$ $\psi_{2}^{\lambda}|=2| \varphi s+s \varphi \mid$. Observe that $\|\varphi s+s \varphi\|=(\varphi s+s \varphi)(s)$ since 
$\|\varphi s+s \varphi\| \leq\|\varphi s\|+\|s \varphi\| \leq 2\|\varphi\|=2 \varphi(\mathbf{1})$ and $\|\varphi s+s \varphi\| \geq \mid(\varphi s+$ $s \varphi)(s) \mid=(\varphi s+s \varphi)(s)=2 \varphi(\mathbf{1})$. By the construction of the absolute value of normal functional [11, the proof of Theorem III.4.2], we have $\left|\psi_{1}^{\lambda}-\psi_{2}^{\lambda}\right|=2|\varphi s+s \varphi|=2 s(\varphi s+s \varphi)=2(\varphi+s \varphi s)$

With the notation $\varepsilon=(\varphi(a)-\varphi(s a s)) / \varphi(a)$, we obtain by a straightforward calculation $\left|\psi_{1}^{\lambda}-\psi_{2}^{\lambda}\right|(a)=2(2-\varepsilon) \varphi(a)$ as well as $\left(\psi_{1}^{\lambda}+\psi_{2}^{\lambda}\right)(a)=$ $\left(\lambda(1-\varepsilon)+\lambda^{-1}\right) \varphi(a)$. Since $\min _{\lambda>0}\left\{\lambda(1-\varepsilon)+\lambda^{-1}\right\}=2 \sqrt{1-\varepsilon}$ and $2-\varepsilon>\sqrt{1-\varepsilon}$ for any $\varepsilon \in(0,1)$, there exists $\lambda_{0}>0$ such that $2(2-\varepsilon)>\lambda_{0}(1-\varepsilon)+\lambda_{0}^{-1}$. Therefore $\left|\psi_{1}^{\lambda_{0}}-\psi_{2}^{\lambda_{0}}\right|(a)>\left(\psi_{1}^{\lambda_{0}}+\psi_{2}^{\lambda_{0}}\right)(a)$.

Theorem 1. For $a \in \mathcal{M}^{+}$the following conditions are equivalent:

(i) a lies in the center $\mathcal{Z}$ of $\mathcal{M}$;

(ii) pap $\leq$ a for each $p \in \mathcal{M}^{\text {pr }}$

(iii) $\varphi^{+}(a) \leq \varphi_{1}\left(\right.$ a) for each $\varphi \in \mathcal{M}_{*}^{h}$ and any decomposition $\varphi=$ $\varphi_{1}-\varphi_{2}$ with $\varphi_{1}, \varphi_{2} \in \mathcal{M}_{*}^{+}$

(iv) the mapping $\varphi \mapsto \varphi^{+}(a)\left(\varphi \in \mathcal{M}_{*}^{h}\right)$ is monotone, i. e., $\varphi, \psi \in \mathcal{M}_{*}^{h}, \varphi \leq \psi$ imply $\varphi^{+}(a) \leq \psi^{+}(a)$;

$(v)$ the mapping $\varphi \mapsto \varphi^{+}(a)\left(\varphi \in \mathcal{M}_{*}^{h}\right)$ is subadditive, i. e., $(\varphi+\psi)^{+}(a) \leq \varphi^{+}(a)+\psi^{+}(a)$ for all $\varphi, \psi \in \mathcal{M}_{*}^{h}$;

(vi) the mapping $\varphi \mapsto|\varphi|(a)\left(\varphi \in \mathcal{M}_{*}^{h}\right)$ is subadditive, i. e., $|\varphi+\psi|(a) \leq|\varphi|(a)+|\psi|(a)$ for all $\varphi, \psi \in \mathcal{M}_{*}^{h}$.

Proof. $(i) \Rightarrow$ (ii) For $a \in \mathcal{Z}^{+}$and $p \in \mathcal{M}^{\text {pr }}$, one has $a=p a p+(\mathbf{1}-$ p)a $(\mathbf{1}-p) \geq$ pap.

(ii) $\Rightarrow($ iii $)$ For $\varphi \in \mathcal{M}_{*}^{h}$, let $p$ be the support projection of $\varphi^{+}$ (see [11, Section III.4]). Then $\varphi^{+}(a)=(p \varphi p)(a) \leq\left(p\left(\varphi+\varphi_{2}\right) p\right)(a)=$ $\left(p \varphi_{1} p\right)(a)=\varphi_{1}($ pap $) \leq \varphi_{1}(a)$.

$($ iii $) \Rightarrow($ iv $)$ This follows from the equality $\varphi=\psi^{+}-\left(\psi^{-}+(\psi-\varphi)\right)$.

$(i v) \Rightarrow(v)$ For $\varphi, \psi \in \mathcal{M}_{*}^{h}$, one has $\varphi \leq \varphi^{+}$and $\psi \leq \psi^{+}$, hence $\varphi+\psi \leq \varphi^{+}+\psi^{+}$. Then the condition $(i v)$ entails $(\varphi+\psi)^{+}(a) \leq$ $\varphi^{+}(a)+\psi^{+}(a)$.

$(v) \Rightarrow(v i)$ By the condition $(v)$, for $\varphi, \psi \in \mathcal{M}_{*}^{h}$, it holds $(\varphi+$ $\psi)^{+}(a) \leq \varphi^{+}(a)+\psi^{+}(a)$. Also, $(\varphi+\psi)^{-}(a)=(-\varphi-\psi)^{+}(a) \leq$ $(-\varphi)^{+}(a)+(-\psi)^{+}(a)=\varphi^{-}(a)+\psi^{-}(a)$. Hence $|\varphi+\psi|(a)=(\varphi+$ $\psi)^{+}(a)+(\varphi+\psi)^{-}(a) \leq \varphi^{+}(a)+\psi^{+}(a)+\varphi^{-}(a)+\psi^{-}(a)=|\varphi|(a)+|\psi|(a)$.

$(v i) \Rightarrow(i)$ If an operator $a \in \mathcal{M}^{+}$satisfies $(v i)$, then it follows from Lemma 1 that $\varphi($ sas $)=\varphi(a)$ for each symmetry $s$ in $\mathcal{M}$ and any $\varphi$ in $\mathcal{M}_{*}^{h}$. Therefore $a=$ sas for each symmetry $s$ in $\mathcal{M}$. As it is easily seen, the latter implies that a commutes with each projection in $\mathcal{M}$ and therefore lies in $\mathcal{Z}$.

Remark 1. Clearly, for $a \in \mathcal{M}^{+},(v)$ is equivalent to the condition 
(vii) the mapping $\varphi \mapsto \varphi^{+}(a)\left(\varphi \in \mathcal{M}_{*}^{h}\right)$ is convex.

As well, $(v i)$ is equivalent to the conditions

(viii) the mapping $\varphi \mapsto|\varphi|(a)\left(\varphi \in \mathcal{M}_{*}^{h}\right)$ is convex.

It follows from Theorem 1 that for $a \in \mathcal{M}^{+}$each of the conditions (vii), (viii) is equivalent to $a \in \mathcal{Z}$.

Corollary 1. For $a \in \mathcal{M}^{+}$, each of the conditions $(i)-($ viii $)$ is equivalent to each of the following conditions:

(ix) $|\varphi|(a)=\left\|a^{\frac{1}{2}} \varphi a^{\frac{1}{2}}\right\|$ for all $\varphi$ in $\mathcal{M}_{*}$;

(x) the mapping $\varphi \mapsto|\varphi|(a)$ is subadditive on $\mathcal{M}_{*}$,

$$
\text { i. e., }|\varphi+\psi|(a) \leq|\varphi|(a)+|\psi|(a) \text { for all } \varphi, \psi \in \mathcal{M}_{*} \text {. }
$$

Proof. Let $a \in \mathcal{Z}^{+}$. For $\varphi \in \mathcal{M}_{*}$, let $\varphi=u|\varphi|$ be the polar decomposition [11, Section III.4]. Then $|\varphi|(a)=\left(u^{*} \varphi\right)(a)=\varphi\left(u^{*} a\right)=$ $\varphi\left(a^{\frac{1}{2}} u^{*} a^{\frac{1}{2}}\right)=\left(a^{\frac{1}{2}} \varphi a^{\frac{1}{2}}\right)\left(u^{*}\right) \leq\left\|a^{\frac{1}{2}} \varphi a^{\frac{1}{2}}\right\|$. On the other hand, $\left\|a^{\frac{1}{2}} \varphi a^{\frac{1}{2}}\right\|=$ $\left\|a^{\frac{1}{2}} u|\varphi| a^{\frac{1}{2}}\right\|=\left\|u a^{\frac{1}{2}}|\varphi| a^{\frac{1}{2}}\right\| \leq\left\|a^{\frac{1}{2}}|\varphi| a^{\frac{1}{2}}\right\|=|\varphi|(a)$. Hence $(i x)$ is satisfied.

$(i x) \Rightarrow(x)$. If $(i x)$ holds true, then we have $|\varphi+\psi|(a)=\| a^{\frac{1}{2}}(\varphi+$ $\psi) a^{\frac{1}{2}}\|\leq\| a^{\frac{1}{2}} \varphi a^{\frac{1}{2}}\|+\| a^{\frac{1}{2}} \psi a^{\frac{1}{2}} \|=|\varphi|(a)+|\psi|(a)$.

Of course, $(x)$ implies $(v i)$.

Remark 2. From the preceding proof, it is seen that we can add the following condition to the list $(i)-(x)$ of equivalent conditions.

(xi) $|\varphi|(a)=\left\|a^{\frac{1}{2}} \varphi a^{\frac{1}{2}}\right\|$ for all $\varphi$ in $\mathcal{M}_{*}^{h}$.

The following theorem is an analog of Gardner's characterization of traces by "triangle inequality" [6] (see, also, [8]).

Theorem 2. A positive element a of $\mathcal{M}$ belongs to the center of $\mathcal{M}$ if and only if the inequality $|\varphi(a)| \leq|\varphi|(a)$ holds for any $\varphi$ in $\mathcal{M}_{*}$.

Proof. Taking into account Corollary 1, for $a \in \mathcal{Z}$ and $\varphi \in \mathcal{M}_{*}$, we get $|\varphi(a)|=\left|\left(a^{\frac{1}{2}} \varphi a^{\frac{1}{2}}\right)(\mathbf{1})\right| \leq\left\|a^{\frac{1}{2}} \varphi a^{\frac{1}{2}}\right\|=|\varphi|(a)$.

Let $|\varphi(a)| \leq|\varphi|(a)$ for any $\varphi$ in $\mathcal{M}_{*}$, then $|(u \psi)(a)|=|\psi(u a)| \leq \psi(a)$ for any unitary $u$ and any positive normal $\psi$. Since the unit ball of $\mathcal{M}$ is the closed convex hull of the set of unitaries [9] (see also [7, 1.1.12]), it follows that $|\psi(x a)| \leq\|x\| \psi(a)$ for every $x$ in $\mathcal{M}$. But then the functional $\psi a$ attains its norm at 1 and is therefore positive [5, 2.1.9]. If $x \in \mathcal{M}^{s a}$ then $\psi(x a)=\overline{\psi(x a)}=\psi(a x)$. It follows that $x a=a x$ for any $x \in \mathcal{M}^{s a}$ and therefore $a$ belongs to the center.

Corollary 2. A positive element a of a $C^{*}$-algebra $\mathcal{A}$ belongs to the center of $\mathcal{A}$ if and only if the inequality $|\varphi(a)| \leq|\varphi|(a)$ holds for any $\varphi$ in $\mathcal{A}^{*}$. 
Proof. Let $(\pi, \mathfrak{H})$ be the universal representation of $\mathcal{A}$ and $\mathcal{M}(\pi)$ be the universal enveloping von Neumann algebra [11, Section III.2]. By construction of $\mathcal{M}(\pi)$, the spaces $\mathcal{A}^{*}$ and $\mathcal{M}(\pi)_{*}$ are isometrically isomorphic in a natural way. For $\varphi \in \mathcal{A}^{*}$, we will denote by $\widetilde{\varphi}$ the corresponding functional in $\mathcal{M}(\pi)_{*}$. Note that $\widetilde{|\varphi|}=|\widetilde{\varphi}|$ by construction of absolute value [5, 12.2.7, 12.2.8].

Let $a$ be a positive element of the center of $\mathcal{A}$. It is easy to see that $\pi(a)$ belongs to the center of $\mathcal{M}(\pi)$. Then for any $\varphi \in \mathcal{A}^{*}$ we have $|\varphi(a)|=|\widetilde{\varphi}(\pi(a))| \leq|\widetilde{\varphi}|(\pi(a))=\widetilde{\varphi}|(\pi(a))=| \varphi \mid(a)$.

On the other hand, let $a$ be a positive element of $\mathcal{A}$ and the inequality $|\varphi(a)| \leq|\varphi|(a)$ hold for any $\varphi$ in $\mathcal{A}^{*}$. Then $|\widetilde{\varphi}(\pi(a))| \leq|\widetilde{\varphi}|(\pi(a))$ for any $\tilde{\varphi}$ in $\mathcal{M}(\pi)_{*}$, which implies that $\pi(a)$ lies in the center of $\mathcal{M}(\pi)$, hence $a$ lies in the center of $\mathcal{A}$.

Remark 3. One can easily adapt the conditions $(i i i)-(x i)$ to the case of $\mathrm{C}^{*}$-algebras and see that each of those modified conditions characterizes central elements.

\section{REFERENCES}

[1] A.M. Bikchentaev, Dokl. Math., 87 (1), 79-82 (2013).

[2] A.M. Bikchentaev, Sib. Math. J., 51 (6), 971-977 (2010).

[3] A.M. Bikchentaev, Math. Notes, 89 (3-4), 461-471 (2011).

[4] Dinh Trung Hoa, O.E. Tikhonov, Math. Notes, 88 (2), 177-182 (2010).

[5] J. Dixmier, $C^{*}$-algebras, (North Holland Publishing co., Amsterdam, 1977).

[6] L.T. Gardner, Can. J. Math. 31, 1322-1328 (1979).

[7] G. K. Pedersen, $C^{*}$-algebras and their automorphism groups, (London Math. Soc., Monographs 14, Academic Press, London/New York, 1979).

[8] G.K. Pedersen, E. Størmer, Can. J. Math. 34, 370-373 (1982).

[9] B. Russo, H.A. Dye, Duke Math. J. 33, 413-416 (1966).

[10] A.I. Stolyarov, O.E. Tikhonov, A.N. Sherstnev, Math.Notes 72 (3), 411-416 (2002).

[11] M. Takesaki, Theory of operator algebras I, ( Encyclopaedia of Mathematical Sciences 124, Reprint of the first (1979) edition, Operator Algebras and Noncommutative Geometry, 5 Springer-Verlag, Berlin, 2002).

[12] O.E. Tikhonov, Positivity, 9 (2), 259-264 (2005). 